\title{
A Preliminary Study on the Effectiveness of the Peer Relationship Enhancement Program in Adolescents at Risk for Internet and Smartphone Addiction
}

\author{
Tae-Ho Kim ${ }^{1,2}$, Young-Soon Jun², Young-Tae Shin ${ }^{3}$, So-Hee Lim ${ }^{3}$, and Joo-Won Seo ${ }^{4}$ \\ ${ }^{1}$ Department of Psychiatry, College of Medicine, Konkuk University, Chungju, Korea \\ ${ }^{2}$ Department of Psychiatry, Konkuk University Chungju Hospital, Chungju, Korea \\ ${ }^{3}$ Department of Psychiatry, Chungbuk National University, Cheongju, Korea \\ ${ }^{4}$ Appletree Psychiatric Clinic, Seoul, Korea
}

Objectives: The purpose of this study is to evaluate the preliminary effects of the Peer Relationship Enhancement Program in adolescents deemed to be in an at-risk group for Internet and smartphone addiction.

Methods: The study group consisted of 33 adolescent participants ( 24 boys and 9 girls) at risk of Internet and smartphone addiction in small and medium-sized cities. The subjects participated in 8 consecutive sessions of the Peer Relationship Enhancement Program. The Korean Internet Addiction Proneness Scale, the Korean Smartphone Addiction Proneness Scale, the Real-Ideal Self Discrepancy Scale, the UCLA Loneliness Scale, the Peer Intimacy Scale, and the Escaping from the Self Scale were evaluated before the initial and after the final session. A paired t-test was performed to statistically analyze the data.

Results: The Peer Relationship Enhancement Program led to a significant decrease $(\mathrm{p}<0.05)$ in self-reported measures of The Korean Internet Addiction Proneness Scale, the Korean Smartphone Addiction Proneness Scale, and the Real-Ideal Self Discrepancy Scale.

Conclusion: The Peer Relationship Enhancement Program reduces the risk of Internet and smartphone addiction and effectively prevents the associated problems.

Key Words: Internet addiction; Smartphone addiction; Adolescent; Peer Relationship Enhancement Program.

Received: August 10, 2017 / Revision: October 13, 2017 / Accepted: October 31, 2017

Address for correspondence: Tae-Ho Kim, Department of Psychiatry, Konkuk University Chungju Hospital, College of Medicine, Konkuk University, 82 Gugwon-daero, Chungju 27376, Korea

Tel: +82-43-840-8990, Fax: +82-43-840-8982, E-mail: taosea@naver.com

\section{INTRODUCTION}

In Korea, use of the Internet and smart technology has become ubiquitous across all age groups due to the popularization of smartphones, increased Internet usage, and diversification of content. The results of an Internet overdependence study conducted by the National Information Society Agency in 2016 found that $17.8 \%$ of the population was in the overdependence risk group, a $1.6 \%$ increase from the previous year. Around $30.6 \%$ of adolescents, the highest proportion among all age groups (infants/children, adults, $60+$ years old), were in this overdependence risk group. ${ }^{1)}$ The most common form of Internet use was instant messaging (94.5\%), followed by games (81.3\%), web surfing (73.7\%), social media (65\%), and news searches (60.6\%). Compared to the normal user group, the overdependence risk group used more diverse forms of

This is an Open Access article distributed under the terms of the Creative Commons Attribution Non-Commercial License (http://creativecommons.org/licenses/by-nc/4.0) which permits unrestricted non-commercial use, distribution, and reproduction in any medium, provided the original work is properly cited. content with a higher utilization rate; the game utilization rate, in particular, was $20.2 \%$ higher than in the normal user group, $97.7 \%$ and $77.7 \%$ respectively. ${ }^{1)}$ There is currently no standardized definition or unified terms related to the concept of Internet addiction, and there are still various opinions among scholars. ${ }^{2}$ This study will discuss Internet addiction based on the broad concept of addiction as argued by Young. ${ }^{3)}$

As the Internet and smartphones have become more common, there is an increasing pattern of personal relations, such as meeting, talking, and playing with friends, taking place in a virtual world rather than through face-to-face encounters. Further, the Internet and smartphones have become convenient means of communication for adolescents. This technology can satisfy their need for self-expression and the Internet is an important cultural space among their peers. As such, Internet use is regarded as a convenient tool of communication for adolescents that can be used freely anywhere and at any time. 
With increased Internet usage, however, negative consequences of overuse are becoming more prevalent. Notably, adolescents are experiencing problems in various aspects of their lives such as physical symptoms, family conflict, difficulties in personal relations, maladjustment to school life, lower grades, and mental health issues as they overuse and become excessively immersed in the Internet. ${ }^{4)}$ Moreover, there has been an increase in cyber bullying occurring online, such as criticizing or ostracizing an individual through mobile messaging, the most powerful means of communication among adolescents. ${ }^{4)}$ With an increase in Internet and smartphone addiction cases that may lead to serious issues with emotional, physical, and personal relation functionalities, there is now more research on the vulnerability factors for this addiction.

High impulsivity and low communication skills were reported factors that influence Internet addiction among adolescents. In studies conducted on middle school students, the tendency for addictive Internet usage was predominantly found where the relationship with parents was less amicable. ${ }^{5,6)}$ In addition, depression, anxiety, temperament, hyperactivity/impulsiveness, low family cohesion and adaptability, and dysfunctional communication within the family were reported to be factors that impact Internet addiction. ${ }^{7-9)}$ Jun et al. ${ }^{10)}$ assessed vulnerability factors in terms of family function and personal relations by researching risk factors related to Internet and smartphone addiction for 1041 middle school students, high school students, and special minority groups (community child centers, child welfare institutions, sunflower centers, shelters, multicultural centers, and hospital clinical groups). From the family function perspective, Internet and smartphone addiction vulnerability factors included the relationship with parents, as well as family adaptability and cohesion. From the personal relations perspective, intimacy in friendships and loneliness served as vulnerability factors. These results are consistent with previous studies that have examined factors impacting Internet addiction.

Adolescents, the main Internet and smartphone user group, are going through a phase that involves many physical, emotional, and psychological changes in development. In addition, friendships during this period are more important to personal development and adjustment to school and society than in any other period in an individual's life. ${ }^{11)}$ Forming normal, positive friendships is an important emotional and cognitive factor in healthy growth and development of adolescents. However, due to a lack of research on the link between peer relationships and addiction to the Internet and smartphones, ${ }^{12)}$ there must be more research focused on examining the relationship of peer groups and addictive use of the Internet and smartphones among adolescents. Lee and
Myung $^{11)}$ found many negative factors of peer relationships in the mobile phone addiction group, such as conflicts, opposition, selfishness, withdrawal, isolation, and dissatisfaction with peer relationships, through research on the degree of mobile phone addiction and usage status among adolescents. In the study by Suh and $\mathrm{Yoo}^{12)}$ which examined the characteristics of Internet addiction and friendships among adolescents, subjects with higher levels of Internet addiction were found to have decreased satisfaction with friendships in their daily life.

Adolescence is a time when social relationships are formed, and having fewer social skills puts you at higher risk for Internet addiction. Adolescents at risk of Internet addiction become immersed in a virtual space for a long time, and are thus unable to form close peer relationships and lack social skills, thereby becoming at higher risk of maladjustment to difficulties. ${ }^{13)}$ Upon examining the results of previous studies, it is necessary to establish a treatment approach and countermeasures that are appropriate for the vulnerability factors of addiction and the characteristics of adolescents, who make up the largest proportion of the Internet and smartphone addiction risk group. Further, while the Internet and smartphones may appear to temporarily relieve stress in adolescents, they do not serve as countermeasures that can help resolve problems. Therefore, it is necessary to develop programs that help prevent Internet and smartphone addiction in order to keep adolescents from experiencing difficulties in their daily lives due to these issues. ${ }^{14)}$ Hence, this study attempts to verify the effects of the Peer Relationship Enhancement Program, developed based on the study by Jun et al., ${ }^{10)}$ on adolescents at risk of Internet and smartphone addiction.

\section{METHODS}

\section{Subjects}

The questionnaire was given to a total of 429 middle school students taking part in the adolescent Internet and smartphone usage status survey and preventative training in a small-to-medium sized city in the Chungcheongbuk-do. Of these students, 33 (24 male and 9 female students) were in the Internet and smartphone addiction risk group and took part in the program. The program was conducted after receiving written consent from the teachers, parents, and adolescents of the participating institution. This study was approved by the Institutional Review Board (2014-041).

\section{Program structure and progress}

The researcher created the Peer Relationship Enhancement Program based on the study by Jun et al., ${ }^{10)}$ with the purpose being to provide opportunities to develop communica- 
tion skills, the ability to control emotions, and social adjustment skills by having students learn and practice useful social skills within and outside the group (Appendix).

The program was conducted once a week for 8 weeks at two middle schools in a small to mid-sized city in the Chungcheongbuk-do from September 8, 2015 to October 27, 2015 and July 11, 2016 to August 29, 2016. One clinical psychologist headed the program, and one researcher also participated in the program. Each session of the program lasted for 50 minutes and involved various games as well as social skill training for improving peer relationships. To assess the degree of change in adolescents through their participation in the program, students were measured on the Internet and smartphone addiction self-diagnosis scale, ideal-reality selfdiscrepancy scale, personal relation loneliness scale, friendship intimacy scale, and the self-escape scale before beginning and at the end of the program.

\section{Measurement tools}

\section{Korean Internet Addiction Proneness Scale}

This scale is a standardized Korean Internet addiction scale that was developed based on the Young scale. ${ }^{3,15)}$ It consists of 15 questions that measure problems, such as daily life disabilities, inclination toward the virtual world, withdrawal, and resistance factors, regarding Internet usage on a 4-point scale. Based on the total score and individual scores for each factor, it is possible to classify the subjects into a high-risk user group showing all symptoms of withdrawal, resistance, and daily life disabilities, a potential-risk user group showing at least one of these symptoms, or a normal user group. ${ }^{15)}$

\section{Korean Smartphone Addiction Proneness Scale}

This scale is a standardized Korean smartphone addiction scale that was developed based on the Internet and smartphone addiction scales. It consists of 15 questions ${ }^{16)}$ that involve details such as daily life disabilities, inclination toward the virtual world, withdrawal, and resistance factors. As with the Korean Internet Addiction Proneness Scale, subjects were divided into a high risk, potential risk, and normal user group based on their total score and individual scores for each factor. ${ }^{16)}$

\section{Ideal-Reality Self-Discrepancy Scale}

This Ideal-Reality Self-Discrepancy Scale was developed through Hoge and McCarthy ${ }^{17)}$ using questions that were adapted and validated by Choi and Lee. ${ }^{18}$ The questions are measured on a 6-point scale involving the actual and ideal self scale that evaluate one's own appearance, popularity, and personality. Consisting of 9 questions, the last of which is open-ended and helps measure individual traits, however this question was excluded from analysis in this study. Idealreality self-discrepancy is the difference in the total scores between the ideal and actual self scales with a range of -40 to +40 points.

\section{Personal Relations Loneliness Scale}

The revised UCLA Loneliness Scale was designed by Russel et al., ${ }^{19)}$ and the questions in this study were adapted and validated by Kim. ${ }^{20)}$ This scale includes 20 questions that measure close and social acquaintances, as well as the sense of belonging on a 4-point scale.

\section{Friendship Intimacy Scale}

This Friendship Intimacy Scale was designed by Ray and Cohen ${ }^{21)}$ and uses questions that were adapted and validated by Lee. ${ }^{22)}$ This scale includes sub-factors, such as support, sense of closeness, fellowship, agreement, and affection and addresses content regarding the behavioral and mental domain of each sub-factor. There are a total of 18 questions measured on a 4-point scale.

\section{Self-Escape Scale}

To examine self-escape tendencies, the Self-Escape Scale, which was created by Shin ${ }^{23}$ based on the theory by Baumeister, ${ }^{24)}$ was used. This scale is composed of 71 questions that include characteristics of a state of cognitive decline, however this study used the abridged scale consisting of 35 questions.

\section{Data analysis}

To verify the effects of the Peer Relationship Enhancement Program in this study, a comparative analysis was performed through a paired t-test between the pre- and post-test. The Statistical Package for the Social Sciences (SPSS) 12.0 (SPSS Inc., Chicago, IL, USA) was used for all collected data.

\section{RESULTS}

\section{Demographic characteristics}

There was a total of 33 students (24 males and 9 females) who participated in the Peer Relationship Enhancement Pro-

Table 1. Demographic characteristics

\begin{tabular}{lc}
\hline \multicolumn{1}{c}{ Variable } & Subject (\%) \\
\hline $\begin{array}{l}\text { Gender } \\
\text { Male }\end{array}$ & $24(72.7)$ \\
Female & $9(27.3)$ \\
Age (year) & \\
14 & $15(45.5)$ \\
15 & $18(54.5)$ \\
\hline
\end{tabular}


Table 2. Pre-treatment and post-treatment outcome variables $(n=33)$

\begin{tabular}{|c|c|c|c|c|}
\hline \multirow{2}{*}{ Variable } & \multicolumn{2}{|c|}{ Mean (SD) } & \multirow{2}{*}{$t$} & \multirow{2}{*}{$\mathrm{p}$} \\
\hline & Pre & Post & & \\
\hline K-Scale & $39.72(4.73)$ & $34.22(7.71)$ & $3.828^{* *}$ & 0.001 \\
\hline S-Scale & $40.06(5.77)$ & $34.61(7.44)$ & $3.938^{* * *}$ & 0.000 \\
\hline Real-ideal self discrepancy & $-6.81(7.23)$ & $-4.13(5.68)$ & $-3.045^{* *}$ & 0.005 \\
\hline Escaping from the self & $12.73(7.50)$ & $12(7.41)$ & 0.938 & 0.355 \\
\hline Peer relationship & $56.16(10.93)$ & $57.53(8.30)$ & -0.836 & 0.410 \\
\hline Loneliness & $55.45(8.97)$ & $56.13(9.72)$ & -0.555 & 0.583 \\
\hline
\end{tabular}

${ }^{* *} \mathrm{p}<0.01,{ }^{* * *} \mathrm{p}<0.001$. K-Scale: Korean Internet Addiction Proneness Scale, S-scale: Korean Smartphone Addiction Proneness Scale

gram. Among them, 15 students were in their first year of middle school, while 18 students were in their 2nd year (Table 1).

\section{Pre- and post-program effects}

The difference in scores in the measurement tools recorded before and after the Peer Relationship Enhancement Program were compared. First, there was a statistically significant decrease in Internet $(\mathrm{t}=3.828, \mathrm{p}<0.01)$ and smartphone use $(t=3.938, p<0.001)$. Moreover, the Peer Relationship Enhancement Program also led to a decrease in the degree of self-discrepancy $(t=-3.045, \mathrm{p}<0.01)$. However, there were no significant changes in the Self-Escape, Friendship, and Personal Relationship Loneliness Scale. These results are not consistent with those of previous studies (Table 2).

\section{DISCUSSION}

To verify the effects of the Peer Relationship Enhancement Program, created by the researcher based on the study by Jun et al., ${ }^{10)}$ this study compared 33 students who were at risk of Internet and smartphone addiction before and after their participation in the program. This study assessed participants through an adolescent Internet and smartphone addiction self-diagnosis scale, ideal-reality self-discrepancy scale, personal relationship loneliness scale, friendship intimacy scale, and self-escape scale. The following results and conclusions were derived as a result.

The results showed a statistically significant decrease in the Internet and smartphone self-diagnosis scale score of students who participated in the Peer Relationship Enhancement Program. This result is consistent with the study by Kwon ${ }^{14)}$ who found that personal relationship skills had a negative influence on game addiction, as well as the study by Lee and $\mathrm{Ahn}^{25}$ who found that adolescents with higher personal relationship skills were less likely to have an Internet addiction. Further, there was a significant improvement in personal relationship skills by effectively using various coping methods for social situations through the Peer Relationship Enhancement Program. This coincides with the purpose of this pro- gram which is to train subjects on how to improve their relationships with peers.

When the average ideal-reality self-discrepancy scales were compared before and after the Peer Relationship Enhancement Program, the average score was significantly lower after the program, implying a decrease in the difference between the subject's ideal and actual self. This result is consistent with the study by Kim and Chang, ${ }^{26)}$ which found that the Internet addiction level was higher with a greater difference between the subject's ideal and actual self. The virtual world is a potentially attractive space for adolescents with higher self-discrepancy, considering that they can forget their unsatisfactory self in realityand instead take on a more attractive, ideal self in this virtual world. ${ }^{27}$ Therefore, by narrowing the gap between the subject's actual self and ideal self through the Peer Relationship Enhancement Program, it is thought that subjects can accept themselves as they are and improve their self-esteem.

There was no statistically significant difference in the selfescape, friendship satisfaction, or personal relationship difficulty scale before and after the program. This is inconsistent with results from Jun et al. ${ }^{10)}$ This may be due to the fact that the Peer Relationship Enhancement Program that was conducted in this study did not fully reflect the relevant domains, thereby resulting in insignificant effects. Previous studies emphasize the need for treatment intervention through a psychosocial approach, particularly the importance of developing psychosocial approach programs for adolescents. ${ }^{28)}$ Therefore, it is necessary to verify the order and structure of this study's Peer Relationship Enhancement Program and reconstruct it into a program with optimal results. The limitations of this study are as follows. First, the sample size of this study was small, and since the study was conducted in a partially restricted region, it cannot be generalized to the entire population. Second, the measurement scales in this study were self-reported measurements rather than a direct evaluation from an expert, hence the data may be lacking in consistency and reliability. Third, there was no control group to compare the actual effects of the Peer Relationship Enhance- 
ment Program, and various uncontrolled variables may have impacted the results. Fourth, while Internet and smartphone usage decreased after the program, there must be a follow-up evaluation to determine if this level of usage is maintained as time passes. Despite these limitations, this study showed a significant effect of the Peer Relationship Enhancement Program, as there were significant changes in the students who took part in the program. Therefore, satisfaction with peer relationships may be an important preventative factor in relation to Internet and smartphone addiction.

Based on the above results, the Peer Relationship Enhancement Program, created based on the study by Jun et al., ${ }^{10)}$ had positive effects on the Internet and smartphone addiction risk group, but the details may require further specialization and refining according to factors such as the developmental stage of the adolescents and academic achievement level. This was a preliminary study that verified the effects of the Peer Relationship Enhancement Program. While it has limitations and follow-up studies are required, we have shown it promotes preventative training programs for the Internet and smartphone addiction risk groups. It is possible to systematically follow-up with results if we can connect the program with schools.

\section{CONCLUSION}

This study consisted of the Peer Relationship Enhancement Program, created by the researcher based on the study by Jun et al., ${ }^{10)}$ targeting an Internet and smartphone addiction risk group, and examined the program's influence on the subject' Internet and smartphone usage level and sociability. The results, which compared scores before and after the program, showed a significant change in Internet and smartphone usage level along with a decrease in the degree of self-discrepancy among subjects. This study is noteworthy in that it provided adolescents at risk of Internet and smartphone addiction with an opportunity to reflect on and receive help for their problems through the Peer Relationship Enhancement Program. This program considered the vulnerability factors of Internet and smartphone addiction that were reported in a previous study. The results demonstrated that even a shortterm and limited intervention is effective, but a follow-up study is necessary in the future for a more long-term perspective.

\section{Acknowledgments}

This study was conducted with support from the mental health technical development project under the Ministry of Health and Welfare (HM14C2238).

\section{Conflicts of Interest}

The authors have no financial conflicts of interest.

\section{REFERENCES}

1) National Information Society Agency. The survey on Internet overdependence. Daegu: Ministry of Science, ICT and Future Planning, National Information Society Agency;2016.

2) Kim EY, Lee YS, Han DH, Suh DS, Kee BS. Temperament and genetic polymorphism in Korean male adolescents with Internet addiction tendency. J Korean Neuropsychiatr Assoc 2006;45:468-475.

3) Young KS. Internet addiction: the emergence of a new clinical disorder. Cyberpsychol Behav 1998;1:237-244.

4) National Youth Policy Institute. A study on the situation of youth cyberbullying and measures to prevent it. Sejong: National Youth Policy Institute;2014.

5) Ann S. A study on the addictive usage of the Internet [dissertation]. Seoul: Yonsei Univ.;2000.

6) Jo C. A study on a relation between the Internet addiction of adolescent and family \& school background [dissertation]. Seoul: Kyung Hee Univ.;2001.

7) Choi WH, Son JW, Kim YR, Lee SI, Shin CJ, Kim SK, et al. A study of covert narcissism in adolescent Internet addiction: relationship to anonymity, presence, interactivity, and achievement motivation. J Korean Acad Child Adolesc Psychiatry 2011;22:103-111.

8) Oh EJ, Choi SW, Lee HK, Park JH, Ahn JH, Choi JE, et al. Relationship between Internet game addiction and psychiatric symptoms in a child and adolescent psychiatric clinic. J Korean Acad Child Adolesc Psychiatry 2014;25:136-141.

9) Bang H, Cho A. Relationship between family function and adolescents' Internet game-related behavior. Korean J Dev Psychol 2003; 16:1-22.

10) Jun YS, Kim TH, Shin YT, Jo S. Differences of psychosocial vulnerability factors between Internet and smartphone addiction groups consisting of children and adolescents in a small to medium-sized city. J Korean Acad Child Adolesc Psychiatry 2016;27:188-195.

11) Lee JS, Myung SY. A study of adolescents' peer relation and school life mal adjustment according to their mobile phone use and addiction. J Korean Home Manage Assoc 2007;25:67-86.

12) Suh JH, Yoo AJ. Adolescent friendships: differences in function, structure, and satisfaction by Internet and real life variables. Korean J Child Stud 2001;22:149-166.

13) Jun CA, Park CO, Lee EG. An exploratory study of the related variables on the Internet addiction of adolescents. Korea J Couns 2008; 9:709-726.

14) Kwon JH. The mediation model verification of interpersonal skills on impulsivity, aggression and game addiction: comparative analysis on school levels. J Korea Soc Comput Inf 2010;15:87-98.

15) National Information Society Agency. Development of Korean Internet addiction proneness scale for youth and adults. Seoul: National Information Society Agency;2011.

16) National Information Society Agency. Development of Korean smartphone addiction proneness scale for youth and adults. Seoul: National Information Society Agency;2011.

17) Hoge DR, McCarthy JD. Issues of validity and reliability in the use of real-ideal discrepancy scores to measure self-regard. J Pers Soc Psychol 1983;44:1048-1055.

18) Choi J, Lee Y. The effects of actual self, ideal self and self-discrepancy on depression. Korean J Clin Psychol 1998;17:69-87.

19) Russell D, Peplau LA, Cutrona CE. The revised UCLA Loneliness Scale: concurrent and discriminant validity evidence. J Pers Soc Psychol 1980;39:472-480.

20) Kim OS. Korean version of the revised UCLA Loneliness Scale: reliability and validity test. J Nurs Acad Soc 1997;27:871-879.

21) Ray GE, Cohen R. Children's friendships expectations for proto- 
typical versus actual best friends. Child Stud J 1996;26:209-227.

22) Lee YO. Children's behavioral and psychological expectations on their friendships [dissertation]. Seoul: Kookmin Univ.;1997.

23) Shin MS. An empirical study of the mechanism of suicide: validation of the scale for escape from the self [dissertation]. Seoul: Yonsei Univ.;1992.

24) Baumeister RF. Suicide as escape from self. Psychol Rev 1990;97: 90-113.

25) Lee HC, Ahn CY. A study on the development and effectiveness of cognitive-behavioral therapy for Internet addiction. Korean J Health
Psychol 2002;7:463-486.

26) Kim DE, Chang SS. The effect of self-discrepancy and the way of stress coping on the Internet addiction. J Stud Guid Couns 2011;29: 89-110.

27) Hyeon EJ, Kim KH. The differences of social support, self-discrepancy, and escaping from the self according to the level of addiction and engagement in Internet gaming of adolescents. Korea J Youth Couns 2015;23:229-246.

28) Lee H, Choi TY. The psychosocial approaches of Internet/game addiction. J Korean Soc Biol Ther Psychiatry 2015;21:65-72. 